\title{
Velocidade de Propagação de Ondas de Ultrassom e Resistividade Elétrica para a Detecção do Ataque por Sulfatos de Origem Interna
}

\author{
Ultrasonic and Electrical Resistivity Tests to Identify Attack by Sulfates of Internal Origin
}

Ana Paula Brandão Capraro ${ }^{1}$ (i) orcid.org/0000-0001-6270-6568

Cristofer Bernardi Scremim ${ }^{2}$ (id) orcid.org/0000-0002-1932-8781

Marcelo Henrique Farias de Medeiros ${ }^{1}$ (D) orcid.org/0000-0003-3112-9715

Natália Santos Polegato ${ }^{1}$ (D) orcid.org/0000-0002-7418-555X

${ }^{1}$ Laboratório de Materiais - LAME - PPGECC/UFPR, Paraná, Brasil,

${ }^{2}$ Laboratório de Materiais - PPGEC/UTFPR, Paraná, Brasil.

E-mail do autor principal: Ana Paula Brandão Capraro anapcapraro@gmail.com

\section{Resumo}

A presença de contaminantes nos constituintes do concreto leva a degradação do material desde suas primeiras idades. O estudo objetiva avaliar a viabilidade do emprego dos ensaios ultrassom e resistividade elétrica na identificação de compostos contaminados por enxofre em duas idades, 28 e 56 dias. Foram moldadas quatro séries de corpos de prova de concreto, sendo uma referência e as demais com substituição parcial do agregado miúdo por sulfato de sódio $(0,74 \% ; 1,48 \% ; 7,44 \%)$. Buscando favorecer a ocorrência das reações de hidratação, optou-se pela permanência dos corpos de prova por 28 dias em câmara úmida. Depois, os materiais foram submetidos a um envelhecimento acelerado, alternando os corpos de prova semanalmente entre uma câmara úmida e uma câmara seca. Os resultados mostraram a validade dos ensaios empregados, sendo constatado que o aumento da contaminação por sulfato de sódio reduziu a resistência à compressão e a velocidade de ondas de ultrassom, e aumentou a resistividade elétrica.

Palavras-Chave: Contaminantes; Concreto; Sulfato de sódio.

\section{Abstract}

The presence of contaminants in the concrete constituents leads to degradation of the material from its earliest ages. The objective of this study was to evaluate the feasibility of the use of ultrasonic and electrical resistivity tests to identify compounds contaminated with sulfur at two ages, 28 and 56 days. Four series of concrete specimens were molded, being one reference and the others with partial replacement of the small aggregate by sodium sulfate $(0.74 \%, 1.48 \%, 7.44 \%)$. Aiming to favor the occurrence of hydration reactions, we chose to stay the specimens for 28 days in a humid chamber. Afterwards, the materials were subjected to accelerated aging, alternating the specimens weekly 
between a humid chamber and a dry chamber. The results showed the validity of the tests used, and it was verified that the increase of sodium sulfate contamination reduced the compressive strength and the ultrasonic wave velocity and increased the electrical resistivity.

Key-words: Contaminants; Concrete. Sodium sulfate.

\section{Introdução}

Prevaleceu por muito tempo a opinião de que o concreto praticamente não necessitava de reparos, devido à sua excelente durabilidade, o que the conduziria a um maior tempo de vida útil (PEREIRA et al., 2014) [1]. Helene e Medeiros (2009) citam que o assunto durabilidade do concreto é algo relativamente novo, uma vez que há poucas décadas esse assunto não era considerado de grande importância [2].

De acordo com Brunetaud et al. [3], o ataque de sulfatos, foco da pesquisa, ocorre devido a uma combinação de dois fatores: a precipitação de produtos secundários de sulfato, potencialmente expansivos, e uma deterioração químico-mecânica. O ataque de sulfatos, tanto interno, como o externo, é uma das deteriorações ambientais mais agressivas ao concreto (GAO et al., 2013) [4] e prejudica seu desempenho em relação à durabilidade das estruturas em condições de serviço.

O ataque de sulfato interno é aquele em que o agente agressivo está presente na massa de concreto de forma distribuída, tendo origem nos materiais empregados na dosagem do concreto, sendo este tipo de ataque o foco do presente estudo. No entanto, dentro do âmbito do ataque por sulfatos, deve-se destacar que é até mais comum que haja ataque de sulfato externo, em que existe um ambiente externo rico em sulfato, conforme os estudos de Brunetaud et al. [5].

Uma possível causa de ataque por sulfatos de origem interna é a contaminação dos agregados por minerais sulfetados, como a pirita e pirrotita, que se oxidam com a presença de água e oxigênio, e reagem com os produtos hidratados do cimento Portland, causando variação dimensional, fissuração, desplacamento, amolecimento e desintegração (CENTURIONE et al. 2003) [6].

As normatizações referentes à utilização de agregados contaminados por sulfetos limitam, em sua grande maioria, o teor de $\mathrm{SO}_{3}$ com relação à massa total dos agregados da amostra. A norma francesa, NF P18-540 de 1997 [7], por exemplo, recomenda a utilização de agregados que possuam no máximo $1 \%$ de $\mathrm{SO}_{3}$ com relação a sua massa total.

Uma vez que há a constatação de materiais contaminados por sulfatos e não há um consenso sobre o um limite máximo a ser empregado que não traga prejuízos à integridade do concreto, vê-se a necessidade de estudos na área que visem à identificação de mudanças comportamentais causadas pelo emprego de diferentes teores de contaminação.

Segundo Souza [8], a disponibilidade de sulfato de sódio no interior do material faz com que ocorram reações tardias à hidratação do cimento. Uma vez que há presença de umidade no composto, a solução agressiva de $\mathrm{NaSO}_{4}$ reage com os compostos hidratados do cimento Portland, como o monossulfoaluminato e o hidróxido de cálcio, formando etringita e gipsita.

Levando em consideração o exposto, o presente trabalho visa avaliar a viabilidade do uso dos ensaios de velocidade de propagação de ondas de ultrassom e resistividade elétrica, na detecção de mudanças geradas na matriz interna de concretos dosados com diferentes teores de contaminação por sulfato de sódio.

\section{Materiais e Métodos}

Para avaliação comportamental foram adotadas quatro séries de estudos, sendo uma delas a referência e outras três com teores de substituição do agregado miúdo por sulfato de sódio, simulando a contaminação do mesmo. Para caracterização dos materiais foram realizados os ensaios de ultrassom, resistividade elétrica e resistência à compressão axial do concreto aos 28 dias de idade, sendo que até este momento os corpos de prova foram mantidos em câmara úmida (UR>95\% e $23 \pm 3^{\circ} \mathrm{C}$ ) para alcançar um estágio avançado de hidratação.

Para aceleração do mecanismo de degradação foi adotada a ciclagem semanal dos materiais, após o período de cura, alternando-os entre uma câmara úmida e uma câmara seca. Aos 56 dias de idade os 
mesmos ensaios foram realizados para análise comparativa entre as séries.

A metodologia de avaliação do processo de ataque por sulfatos com a proposta de aceleração do processo por meio de ciclos de umedecimento e secagem já foi utilizada por outros autores, como: Gao et al. [4], Gong et al. [9], Jiang e Niu [10] e Capraro et al. [11]. Para justificar esse procedimento é possível citar o caso de um concreto exposto a ambiente marinho, os ciclos de umedecimento e secagem podem acelerar a deterioração do concreto, assim como nas zonas de respingo de maré.

Para a realização dos ensaios citados foram moldados corpos de prova cilíndricos, $(10 \times 20) \mathrm{cm}$, seguindo as recomendações da NBR 5738 de 2003 [12]. Foram considerados cinco corpos de prova para cada série, em cada idade estudada (28 e 56 dias). Os ensaios de resistividade elétrica e ultrassom, por serem não destrutivos, foram realizados previamente nos corpos de prova que seriam submetidos ao ensaio de compressão axial.

$\mathrm{Na}$ produção dos corpos de prova foi empregado como aglomerante o cimento Portland CP V - ARI, com massa específica igual a $3,14 \mathrm{~g} / \mathrm{cm}^{3}$. Como a pesquisa analisa um mecanismo com reações químicas, esse material foi escolhido por ser, dentre as opções ofertadas na região sul do país, o mais puro, ou seja, o que possui menor porcentagem de adições. A caracterização química, mecânica e física do cimento empregado está disposta na Tabela 1.

O agregado miúdo empregado é uma areia natural, proveniente da região de São Luiz do Purunã, Paraná, com massa específica igual a $2,38 \mathrm{~g} / \mathrm{cm}^{3}$. O agregado graúdo utilizado foi uma brita 01 proveniente da região de Curitiba com massa específica de 2,72 $\mathrm{g} / \mathrm{cm}^{3}$.

Nenhum dos agregados empregados possui, em sua composição, a contaminação natural de enxofre. Dessa forma, optou-se pela contaminação artificial do agregado miúdo, substituindo parte de sua massa por sulfato de sódio anidro. O sulfato de sódio anidro $\left(\mathrm{NaSO}_{4}\right)$, segundo informações do fornecedor, possui massa específica de $2,70 \mathrm{~g} / \mathrm{cm}^{3}$.

Ao todo, foram determinados 4 teores de substituição de $\mathrm{NaSO}_{4}$, com relação à massa total de agregados da amostra (graúdo + miúdo).
O traço adotado como referência (fck de $30 \mathrm{MPa}$ ), empregado em uma usina de concreto da região, foi 1 : 2,70 : 3:20 com relação água/cimento de 0,58, com $1,0 \%$ de aditivo superplastificante para abatimento do tronco de cone de $100 \pm 20 \mathrm{~mm}$. O aditivo superplastificante utilizado foi o Eucon $®$ PL310, que, segundo as especificações do fabricante, possui $\mathrm{pH}$ de 6,5 , massa específica de $1,180 \mathrm{~g} / \mathrm{cm}^{3}$ e não contém cloretos em sua composição.

Optou-se neste estudo por simular a presença do sulfato no agregado do concreto, desse modo, foi feita a substituição em massa de parte do agregado miúdo por sulfato de sódio. Como os materiais a serem substituídos possuem massas específicas distintas (agregado miúdo a ser substituído por $\mathrm{NaSO}_{4}$ ), optouse pela adaptação do traço, em massa, a cada teor avaliado, com o intuito de manter o consumo de cimento fixo em $309,7 \mathrm{~kg} / \mathrm{m}^{3}$.

Os teores de contaminação foram adotados com base nas referências normativas existentes sobre 0 assunto. A norma francesa, NF P18-540 [7], por exemplo, permite a utilização de agregados contaminados, desde que o teor de $\mathrm{SO}_{3}$ não ultrapasse $1 \%$ da massa total de agregados. Já para o Instituto Americano de Concreto, 201.2R-01 de 1991 [13], o limite de $\mathrm{SO}_{3}$ estabelecido é de 0,5\%.

No presente artigo, foram adotados 4 teores de $\mathrm{SO}_{3}$, sendo dois teores considerados abaixo do limite estabelecido pelo ACI e 2 teores acima. Em relação à norma francesa, apenas um dos teores é considerado elevado.

Os teores empregados de $\mathrm{NaSO}_{4}$ foram obtidos a partir das porcentagens em $\mathrm{SO}_{3}$ multiplicadas por 2 fatores de conversão, sendo eles: equivalência de enxofre entre o $\mathrm{SO}_{3}$ e o $\mathrm{SO}_{4}$ e quantidade de $\mathrm{SO}_{4}$ presente no sulfato de sódio anidro $\left(\mathrm{NaSO}_{4}\right)$. Os teores empregados no estudo estão dispostos na Tabela 2. 
Tabela 1: Caracterização do cimento.

\begin{tabular}{|c|c|c|c|c|c|c|c|c|c|c|c|c|}
\hline \multicolumn{13}{|c|}{ Análise química } \\
\hline $\begin{array}{l}\mathrm{CaO} \\
(\%)\end{array}$ & $\begin{array}{l}\mathrm{SiO}_{2} \\
(\%)\end{array}$ & $\begin{array}{l}\mathrm{Al}_{2} \mathrm{O}_{3} \\
(\%)\end{array}$ & $\begin{array}{c}\mathrm{Fe}_{2} \mathrm{O}_{3} \\
(\%)\end{array}$ & $\begin{array}{l}\mathrm{MgO} \\
(\%)\end{array}$ & $\begin{array}{l}\mathrm{SO}_{3} \\
(\%)\end{array}$ & \multicolumn{2}{|c|}{$\begin{array}{c}\text { CaO livre } \\
(\%)\end{array}$} & $\begin{array}{l}\text { P.F. } \\
(\%)\end{array}$ & \multicolumn{2}{|c|}{$\begin{array}{c}\text { Resíduo } \\
\text { Insolúvel (\%) }\end{array}$} & \multicolumn{2}{|c|}{$\begin{array}{l}\text { Equivalente } \\
\text { Alcalino (\%) }\end{array}$} \\
\hline 60 & 18,4 & 4,2 & 2,6 & 4,4 & 2,8 & \multicolumn{2}{|c|}{0,7} & 2,9 & \multicolumn{2}{|c|}{0,7} & \multicolumn{2}{|c|}{0,6} \\
\hline \multicolumn{9}{|c|}{ Análise física } & \multicolumn{4}{|c|}{ Análise mecânica (MPa) } \\
\hline \multicolumn{2}{|c|}{$\begin{array}{l}\text { Expansão } \\
\text { Quente } \\
(\mathrm{mm})\end{array}$} & $\begin{array}{c}\text { Início } \\
\text { de } \\
\text { pega } \\
\text { (h) }\end{array}$ & $\begin{array}{c}\text { Fim de } \\
\text { pega } \\
\text { (h) }\end{array}$ & $\begin{array}{c}\text { Consist. } \\
\text { Normal } \\
(\%)\end{array}$ & \multicolumn{2}{|c|}{$\begin{array}{l}\text { Blaine } \\
\left(\mathrm{cm}^{2} / \mathrm{g}\right)\end{array}$} & $\begin{array}{c}\# 200 \\
(\%)\end{array}$ & $\begin{array}{c}\# 325 \\
(\%)\end{array}$ & $\begin{array}{c}1 \\
\text { dia }\end{array}$ & $\begin{array}{c}3 \\
\text { dias }\end{array}$ & $\begin{array}{c}7 \\
\text { dias }\end{array}$ & $\begin{array}{l}28 \\
\text { dias }\end{array}$ \\
\hline \multicolumn{2}{|c|}{0,5} & $02: 20$ & 03:00 & 28 & \multicolumn{2}{|c|}{4,1} & 0,4 & 3,6 & 22,5 & 34,8 & 42 & 48,8 \\
\hline
\end{tabular}

Fonte: Autores (2017).

Tabela 2: Teores de Equivalentes.

\begin{tabular}{l|l|l}
\hline $\mathbf{\% ~ S O}_{\mathbf{3}}$ & $\mathbf{\% ~ S O}_{\mathbf{4}}$ & $\mathbf{\%} \mathbf{N a S O}_{\mathbf{4}}$ \\
\hline 0,000 & 0,000 & 0,000 \\
0,500 & 0,600 & 0,738 \\
1,000 & 1,200 & 1,476 \\
5,000 & 6,000 & 7,437 \\
\hline
\end{tabular}

Fonte: Autores (2017).

Portanto, são apresentados na Tabela 3, os quatro traços estudados nesta pesquisa.

Tabela 3: Teores de Contaminação e Proporções Estudadas

\begin{tabular}{c|c|c|c|c|c|c}
\hline $\begin{array}{c}\text { \% } \\
\mathbf{N a S O}_{4}\end{array}$ & $\mathbf{a / c}$ & $\begin{array}{c}\text { CP V } \\
\text { ARI } \\
(\mathbf{k g})\end{array}$ & $\begin{array}{c}\text { Sulfato } \\
\mathbf{d e} \\
\text { Sódio } \\
(\mathbf{g})\end{array}$ & $\begin{array}{c}\text { Areia } \\
\mathbf{( k g )}\end{array}$ & $\begin{array}{c}\text { Brita } \\
\mathbf{0 1} \\
\mathbf{( k g )}\end{array}$ & $\begin{array}{c}\text { Consumo } \\
\mathbf{d e} \\
\text { cimento } \\
\left(\mathbf{k g} / \mathbf{m}^{\mathbf{3}} \mathbf{)}\right.\end{array}$ \\
\hline 0 & 0,58 & 1,00 & - & 2,70 & 3,20 & 309,70 \\
0,74 & 0,58 & 1,00 & 43,58 & 2,66 & 3,20 & 309,70 \\
1,48 & 0,58 & 1,00 & 87,47 & 2,62 & 3,20 & 309,70 \\
7,44 & 0,58 & 1,00 & 442,72 & 2,31 & 3,20 & 309,70 \\
\hline
\end{tabular}

Fonte: Autores (2017).

\subsection{Velocidade de Propagação de Ultrassom}

O objetivo do ensaio foi determinação da velocidade de propagação de ondas dos 40 corpos de provas ensaiados, a fim de detectar as variações no concreto ao longo do tempo, devido ao ataque interno por sulfatos.
O ensaio foi executado de acordo com a NBR 8802 de 2013 [14]. Para que se garantisse que as superfícies estivessem secas e planas para a realização do ensaio, os mesmos foram retirados da água 0,5 horas antes de serem ensaiados $e$ regularizados com lixa.

Para permitir o acoplamento entre as faces dos transdutores e o concreto foi aplicada uma fina camada de gel. Logo após, os transdutores foram posicionados em faces opostas dos corpos de prova.

Assim, para cada idade do concreto, o resultado final deu-se pela média das leituras de velocidade nos 5 corpos de prova ensaiados.

\subsection{Resistividade Elétrica (Método Wenner)}

A técnica empregada consistiu no posicionamento de quatro eletrodos na superfície dos corpos de prova de concreto. Os mesmos permaneceram alinhados e equidistantes um do outro.

Como o ensaio é realizado em corpos cilíndricos de $(10 \times 20) \mathrm{cm}$ foi aplicado um coeficiente (fator de forma) de 0,377 , valor este recomendado pela norma espanhola, UNE 83988-2 de 2012 [15].

Para o ensaio de resistividade elétrica foram consideradas válidas as leituras obtidas quando os valores individuais não diferiram da média em mais de $10 \%$.

Assim, o resultado final deu-se pela média calculada dos valores válidos de resistividade elétrica dos 5 corpos de prova ensaiados para cada idade do concreto. 


\subsection{Resistência à Compressão Axial}

Após a realização dos dois ensaios não destrutivos, os corpos de prova cilíndricos foram ensaiados à compressão axial de acordo com a NBR 5739 de 2007 [16].

Antes da realização do ensaio, os corpos de prova foram preparados e capeados com pasta de enxofre.

A máquina de ensaio utilizada foi uma prensa Dinatest, utilizando uma velocidade de carregamento na faixa de 0,45 $\pm 0,15 \mathrm{MPa} / \mathrm{s}$, como estabelece a NBR 5739 de 2007 [16].

\section{Resultados e Discussão}

Para cada traço, em cada idade, foram avaliados 5 corpos de prova. Com a finalidade de eliminar valores espúrios, os resultados obtidos com os ensaios propostos no programa experimental foram tratados estatisticamente por meio do teste de Tukey.

\subsection{Estado Fresco}

A caracterização dos traços no estado fresco contemplou os ensaios de massa específica e slump test.

Como descrito anteriormente, o abatimento do troco de cone foi fixado em $(100 \pm 20) \mathrm{mm}$, desta forma, os quatro traços apresentaram abatimentos iguais a: $80 \mathrm{~mm}$ (referência), $120 \mathrm{~mm}\left(0,5 \% \mathrm{SO}_{3}\right)$; $90 \mathrm{~mm}\left(1,0 \% \quad \mathrm{SO}_{3}\right)$ e $80 \mathrm{~mm}\left(5,0 \% \quad \mathrm{SO}_{3}\right)$, como apresentado na Figura 1.

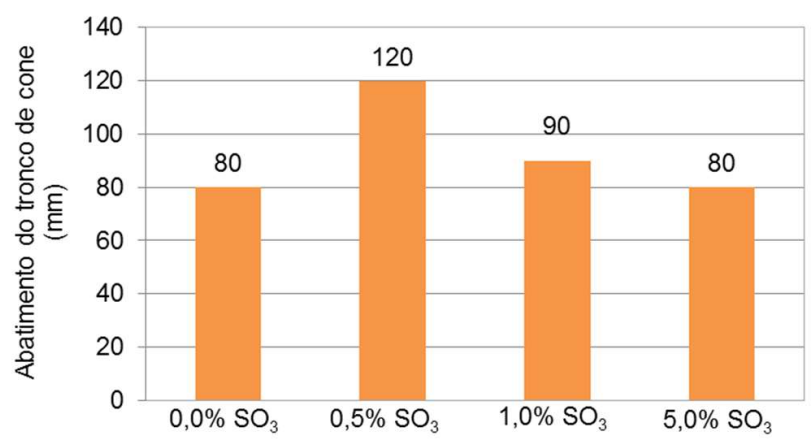

Figura 1: Abatimento do tronco de cone para as séries estudadas.

Fonte: Autores (2017).
A substituição parcial de areia por $0,5 \%$ de sulfato de sódio, nos diferentes teores, aumentou ou manteve o abatimento do tronco. Observou-se que o aumento de fluidez não evoluiu à medida que os maiores valores de substituição foram adotados, regredindo, no traço de 5,0\% de $\mathrm{SO}_{3}$, para um abatimento de 80 $\mathrm{mm}$, igual à referência. Contudo, não se observou grande mudança comportamental, ficando todos os traços compreendidos nesse intervalo com a adição de $1 \%$ de superplastificante.

Quanto à massa específica no estado fresco, não foi encontrada notória diferença entre os traços, uma vez que os materiais substituídos, areia e sulfato de sódio, apresentam massas específicas semelhantes, $2,38 \mathrm{~g} / \mathrm{cm}^{3}$ e $2,70 \mathrm{~g} / \mathrm{cm}^{3}$, respectivamente. Os valores obtidos no ensaio, compreendidos entre $2280 \mathrm{~kg} / \mathrm{m}^{3}$ a 2415 kg/m³ , estão apresentados na Figura 2.

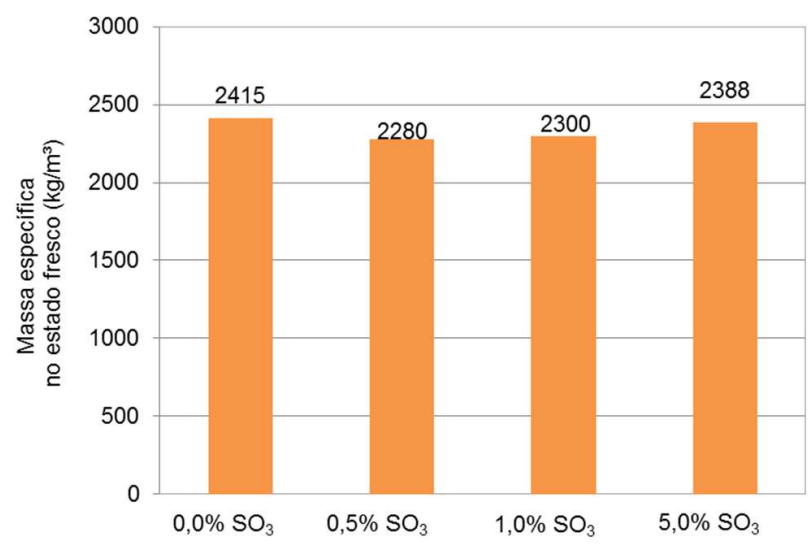

Figura 2: Massa específica no estado fresco para as séries estudadas.

Fonte: Autores (2017).

\subsection{Estado Endurecido}

O primeiro parâmetro avaliado no estado endurecido foi a velocidade de propagação de ondas ultrassônicas, que apontou, aos 28 dias, valores menores para os traços contaminados, quando estes foram comparados com o referência (Figura 3). Com relação ao teste estatístico empregado, foi possível indicar equivalência estatística somente entre os traços de $0,5 \%$ e $1,0 \%$ de contaminação, os demais são considerados estatisticamente diferentes.

Aos 56 dias de idade houve também um aumento da velocidade de propagação das ondas no interior dos materiais (Figura 3), sendo, para todos os traços, um ganho significativo estatisticamente. Entre os grupos http: / / dx.doi.org/10.25286/repa.v2i3.684 


\section{Velocidade de Propagação de Ondas de Ultrassom e Resistividade Elétrica para a Detecção do}

Ataque por Sulfatos de Origem Interna

foi possível notar equivalência entre os traços referência e 0,50\% e entre os traços $0,5 \%$ e $1,0 \%$.

Como já mencionado, as velocidades de ultrassom obtidas neste estudo apontam menores resultados para as séries contaminadas. O mesmo não ocorreu nos estudos de Pereira [17] e Capraro et al. [18], em que, nas mesmas idades estudadas (até os 84 dias), os concretos contaminados por sulfato (pirita) obtiveram velocidades semelhantes ou superiores ao traço referência.

A queda da velocidade de propagação indica o surgimento de espaços vazios no interior da amostra, podendo estes, serem decorrentes de fissuras causadas pelas contaminações empregadas nos traços.

Os resultados de resistividade elétrica dos materiais, parâmetro analisado na sequência, estão apresentados na Figura 4. Observou-se, aos 28 dias, que o traço referência, com $0,0 \%$ de contaminação, apresentou os valores mais baixos de resistividade. Quando feito o estudo de equivalência estatística pelo teste de Tukey, notou-se diferença comportamental somente neste traço, sendo os demais estatisticamente equivalentes $(0,5 \% ; 1,0 \%$ e $5,0 \%)$.

Como a propriedade de resistividade elétrica está ligada à dificuldade de passagem de corrente elétrica em um composto, pode-se afirmar que essa dificuldade foi maior nos concretos contaminados. no interior do concreto. Isso vai de acordo ao exposto por Biczók [19] e Ouyang et al. [20], que, estudando o ataque externo por sulfato de sódio, chegaram a resultados que demonstram o preenchimento dos poros por cristais formados com as reações de sulfatos, aumentando sua compacidade. No entanto, o resultado de ultrassom, apresentado anteriormente, indica o aumento dos espaços vazios presentes na amostra, sugerindo, assim, o aumento da resistividade por efeito químico, e não físico, ou seja, provavelmente a solução presente nos poros dos concretos contaminados ajudou para que estes se tornassem mais resistivos.

Aos 56 dias de idade houve considerável aumento na resistividade dos concretos, sendo este apontado como significativo para todos os traços no teste estatístico. Analisando comparativamente os resultados entre os grupos, nesta última idade, notase comportamento estatisticamente diferente para todos os grupos, sendo o grupo referência o de menor resistividade e o grupo com maior porcentagem de contaminação $(5,0 \%)$ o de maior resistividade.

Quanto à resistência à compressão axial, observou-se que, já aos 28 dias de idade, o traço com maior contaminação $(5,0 \%)$ apresentou o menor valor. Por meio do teste estatístico, este foi o único grupo que pode ser considerado estatisticamente diferente dos demais. A Figura 5 apresenta os valores obtidos nesta idade.

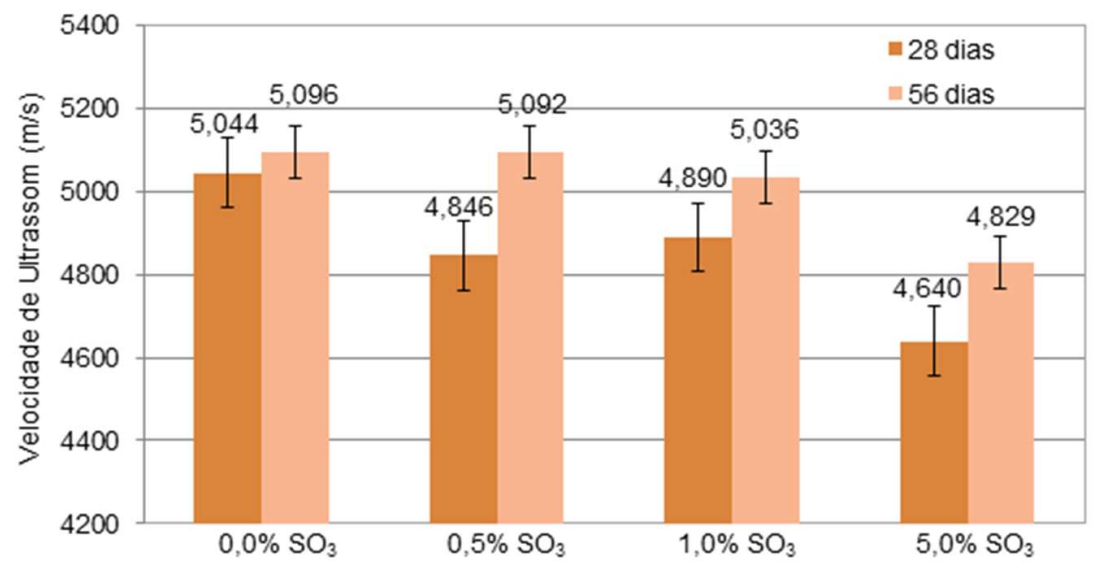

Figura 3: Velocidade de propagação de ondas ultrassônicas nos concretos aos 28 dias de idade (condição de cura em câmara Fonte: Autores (2017) úmida) e 56 dias (condição de ciclagem semanal)

Esse impedimento de passagem de corrente poderia ser explicado pelo preenchimento dos poros 


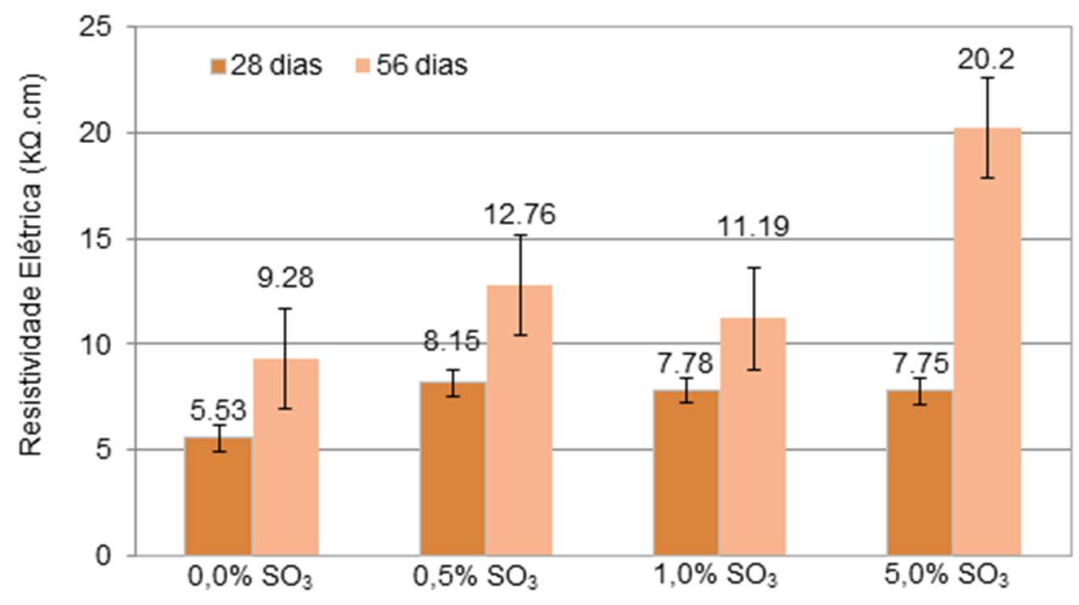

Figura 4: Resistividade elétrica dos traços aos 28 dias de idade (condição de cura em câmara úmida) e aos 56 dias Fonte: Autores (2017). (condição de ciclagem semanal)

Aos 56 dias só foi notado ganho de resistência significativo (28x56dias) para o traço com $0,5 \%$ de contaminação, os demais não aumentaram nem diminuíram a resistência.

Nesta idade os grupos referência e 0,5\% de contaminação são considerados estatisticamente equivalentes, assim como os grupos $1,0 \%$ e 5,0\% de contaminação. Os demais indicaram diferença estatística, como pode ser observado na Figura 5.
Os resultados de resistência à compressão axial, como os de velocidade de ultrassom, também indicam um comportamento diferente do mencionado pela literatura. Neste trabalho a série de maior contaminação apresentou valores menores, quando comparado aos demais, nas duas idades estudadas. Outros trabalhos relacionados com o tema (ARAÚJO, [21]; BICSOK, [19]; OUYANG et al., [20]; CAPRARO, [22]) indicam que para a idade avaliada neste estudo, há um comportamento de aumento da

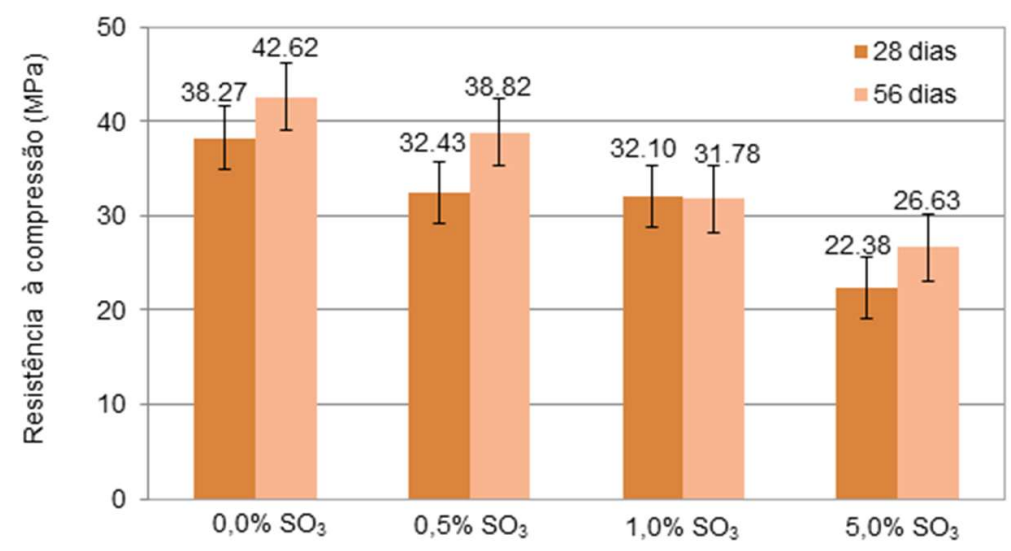

Figura 5: Resistência à compressão axial aos 28 dias de idade (condição de cura em câmara úmida) e aos 56 dias (condição de ciclagem semanal)

Fonte: Autores (2017). 
resistência para concretos contaminados por sulfato, justificado pela colmatação dos espaços vazios.

Uma possível explicação para os dois ensaios, velocidade de ultrassom e resistência à compressão axial, estaria ligada a disponibilização do contaminante para as reações. Biczók, [24] e Ouyang et al. [25] trabalharam com ataque externo de sulfatos com soluções de sulfato de sódio. Já Araújo [26] e Capraro [27] trabalharam com agregados contaminados por sulfetos, não sendo feita a adição pura do contaminante. Assim sendo, acredita-se que a adição de sulfato de sódio anidro foi mais agressiva que as demais, pela disponibilização do material no interior do composto.

\section{Correlação entre os Resultados}

De posse dos resultados obtidos nos ensaios foi realizado o estudo da correlação existente entre eles, isto é, a análise de semelhança de comportamento, queda ou aumento, dos resultados dos ensaios ao longo do tempo.

A correlação entre os ensaios de resistividade elétrica e resistência à compressão mostrou-se fraca, com $\mathrm{R}^{2}=0,30$ para os 28 dias e $\mathrm{R}^{2}=0,58$ para os 56 dias.

A correlação entre os ensaios de ultrassom e resistência à compressão (Figura 6) foi mais satisfatória, quando comparado à correlação entre os ensaios de resistividade elétrica e resistência à compressão. Foi possível verificar que os resultados obtidos nos ensaios são diretamente proporcionais, ou seja, há um aumento de resistência à compressão em ambas as idades, bem como o aumento de velocidade de propagação de ultrassom.

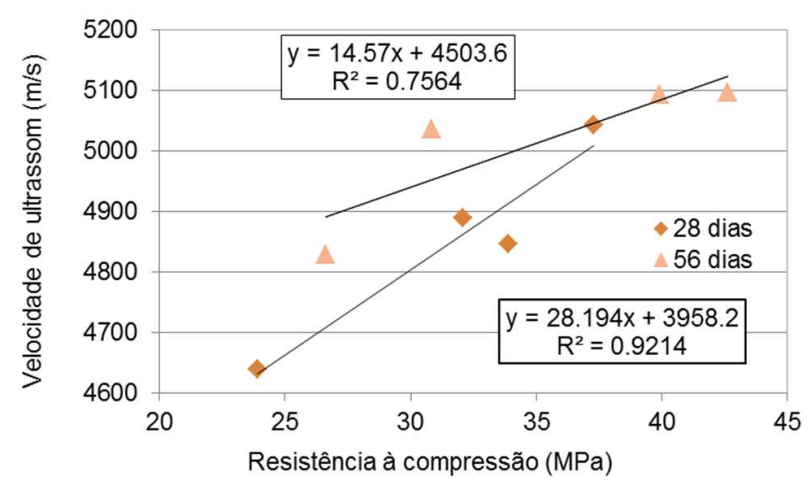

Figura 6: Correlação entre os ensaios de ultrassom e resistência à compressão axial nos corpos de prova aos 28 45 dias (condição de cura em câmara úmida) e 56 dias (condição de ciclagem semanal).

Fonte: Autores (2017).

\section{Conclusões}

O trabalho experimental desenvolvido neste artigo permite realizar as conclusões listadas a seguir para as idades de 28 e 56 dias tratando-se de concreto contaminado por sulfato de sódio:

A contaminação por sulfato de sódio tendeu a aumentar a resistividade elétrica do concreto, provavelmente pelo efeito químico da solução presente nos poros, que deve ser avaliada em estudos futuros para validação;

A contaminação por sulfato de sódio tendeu a reduzir a velocidade de propagação de pulso de ultrassom. Considerando que não ocorreram fissuras visíveis em nenhum dos casos, provavelmente esta redução da velocidade se deve ao surgimento de microfissuras no concreto provocadas pelo ataque por sulfatos incipiente;

O aumento da contaminação por sulfato de sódio tendeu a reduzir a resistência à compressão do concreto, explicado também pelo surgimento de micro fissuras nos concretos contaminados por sulfatos;

O aumento da idade dos corpos de prova, quando comparada a série com ela mesma, resultou na elevação da resistência à compressão, da resistividade elétrica e da velocidade de pulso de ultrassom;

O tempo de exposição empregado neste trabalho foi curto e o processo de degradação por sulfato de sódio necessita de mais tempo para progredir e produzir manifestações patológicas visíveis.

\section{Agradecimentos}

Os autores agradecem ao Laboratório de Materiais e Estruturas da Universidade Federal do Paraná (LAME-DCC-UFPR), ao Programa de Pós-graduação em Engenharia de Construção Civil (PPGECC-UFPR), a Coordenação de Aperfeiçoamento de Pessoal de Nível Superior (CAPES) e Conselho Nacional de Desenvolvimento Científico e Tecnológico (CNPq).

\section{Referências}

[1] PEREIRA, E.; BRAGANÇA, M. O. G. P.; OLIVEIRA, I. C.; GODKE, B.; PORTELLA, K.F. Ataque interno por sulfatos em estruturas de 
concreto contaminadas por pirita: Uma revisão da literatura. $1^{0}$ Congresso Brasileiro de Patologias das Construções. Foz do Iguaçu, Paraná. 2014.

[2] HELENE, P.; MEDEIROS, M. F. Durabilidade e proteção do concreto armado. Revista Téchne. Edição 151, 2009.

[3] BRUNETAUD, X.; DIVET, L.; DAMIDOT, D. Impact of unrestrained Delayed Ettringite Formation-induced expansion on concrete mechanical properties, Cement and Concrete Research, Volume 38, Issue 11, November 2008, Pages 1343-1348.

[4] GAO, J.; YU, Z.; SONG, L.; WANG, T.; WEI, S. Durability of concrete exposed to sulfate attack under flexural loading and drying-wetting cycles, Construction and Building Materials, Volume 39, February 2013, Pages 33-38.

[5] BRUNETAUD, X.; KHELIFA, M.; AL-MUKHTAR, $M$. Size effect of concrete samples on the kinetics of external sulfate attack, Cement and Concrete Composites, Volume 34, Issue 3, March 2012, Pages 370-376.

[6] CENTURIONE, S. L.; KIHARA, Y.; BATTAGIN, A. $F$. Durabilidade de concreto submetidoa ataques de íons sulfato. Anais do $47^{\circ}$ Congresso Brasileiro de Cerâmica. João Pessoa, 2003.

[7] ASSOCIATION FRANÇAISE DE NORMALISATION. XP P18-540. Granulats Définitions, conformité, spécifications. Paris, 1997.

[8] SOUZA, R. B. de. Suscetibilidade de pastas de cimento ao ataque por sulfatos - método de ensaio acelerado. São Paulo, 2006. 131 f. Dissertação (Mestrado em Engenharia) - Escola Politécnica da Universidade de São Paulo.

[9] GONG, J.; CAO, J.; WANG, Y. Effects of sulfate attack and dry-wet circulation on creep of fly-ash slag concrete, Construction and Building Materials, Volume 125, 30 October 2016, Pages 12-20.
[10] JIANG, L.; NIU, D. Study of deterioration of concrete exposed to different types of sulfate solutions under drying-wetting cycles, Construction and Building Materials, Volume 117, 1 August 2016, Pages 88-98.

[11] CAPRARO, A. P. B.; MEDEIROS, M. H. F.; HOPPE FILHO, J.; BRAGANÇA, M. O. G. P.; PORTELLA, K. F; OLIVEIRA, I. C. Reação sulfática interna (RSI) como degradação de matriz cimentícia: comportamento de pastas dosadas com diferentes teores de contaminação por sulfato. Revista ALCONPAT. Vol.7, pp 119-134, 2017.

[12] ASSOCIAÇÃO BRASILEIRA DE NORMAS TÉCNICAS. Concreto - Procedimento para moldagem e cura de corpos de prova. NBR 5738. Rio de Janeiro, 2003.

[13] AMERICAN CONCRETE INSTITUTE. Guide to Durable Concrete. Detroit - USA, 1991 (ACI 201).

[14] ASSOCIAÇÃO BRASILEIRA DE NORMAS TÉCNICAS. Concreto endurecido - Determinação da velocidade de propagação de onda ultra-sônica. NBR 8802. Rio de Janeiro, 2013.

[15] COMITÉS TÉCNICOS DE NORMALIZACIÓN. UNE 83988-2. Durabilidad del hormigón determinación de la resistividad - Parte 2: Método de las cuatro puntas o de Wenner. Norma espanhola (2012).

[16] ASSOCIAÇÃO BRASILEIRA DE NORMAS TÉCNICAS. Concreto - Ensaios de compressão de corpos de prova cilíndricos. NBR 5739. Rio de Janeiro, 2007.

[17] PEREIRA, E. Investigação e monitoramento do ataque por sulfatos de origem interna em concretos nas primeiras idades. Tese de Doutorado apresentada a Universidade Federal do Paraná. Curitiba, 2015.

[18] CAPRARO, A. P.B.; FRANDOLOSO, J. C. P.; MEDEIROS, M. H. F.; PORTELLA, M. D. G.; http: / / dx.doi.org/10.25286/repa.v2i3.684 
PORTELLA, F. F.; GODKE, B.; OLIVEIRA, I. Avaliação de ensaios não destrutivos para a deteç̧ão de concretos dosados com diferentes teores de contaminação por íons sulfeto. Simpósio Paranaense de Patologia das Construções. Curitiba, 2017.

[19] BICZOK, I. Concrete Corrosion and Concrete Protection. Chemical Publishing Company, Inc., New York, 1972.

[20] OUYANG, W.; CHEN, J.; JIANG, M. Evolution of surface hardness of concrete under sulfate attack. Construction and Building Materials, Vol. 53, p. $419-424,2014$.

[21] ARAÚJO, G. S. La reacción sulfática de origen interno em presas de hormigón. Propuesta metodológica de análisis. Tesis Doctoral Universitat Politácnica de Catalunya. Departament d’Enginyeria de la Construcción. Barcelona, 2008.

[22] CAPRARO, A. P. B. Mudanças nas propriedades microestruturais e mecânicas de pastas e argamassas dosadas com diferentes teores de contaminação por sulfetos. Dissertação de mestrado Universidade Federal do Paraná. Curitiba, 2016. 